\title{
Validação de um método analítico rápido por CLAE-UV para determinação de cumarina em guaco (Mikania glomerata Sprengel) confirmado com espectrometria de massas
}

\begin{abstract}
BERTOLDI, F.C. ${ }^{1 *}$; DESCHAMPS, F.C.'; SILVA JUNIOR, A.A.'; CORREA, A.F.'; FRANCO, M.F. ${ }^{2,3}$, EBERLIN, M.N. ${ }^{3}$

'Empresa de Pesquisa Agropecuária e Extensão Rural de Santa Catarina Estação Experimental de Itajaí,, CEP 88318-112, Itajaí - SC, Brasil. 2Instituto de Criminalística, Superintendência da Polícia Técnico-Científica, CEP 05507-06, São Paulo - SP, Brasil. 'Universidade de Campinas, Instituto de Química, CEP 13083-970, Campinas - SP, Brasil. *Autor para correspondência: fabianobertoldi@epagri.sc.gov.br
\end{abstract}

RESUMO: A espécie Mikania glomerata Sprengel, popularmente conhecida no Brasil como guaco, é amplamente utilizada como expectorante para tratar doenças respiratórias e tem a sua atividade farmacológica atribuída principalmente a cumarina. Os resultados mostraram que o método apresenta linearidade de 0,05 a $0,8 \mathrm{mg} \mathrm{mL}^{-1}$. Ele foi considerado seletivo, exato e preciso. A proposta de um método rápido para determinação de cumarina em extratos de guaco torna-se interessante para a rotina de controle de qualidade industrial, visando à obtenção de medicamentos fitoterápicos padronizados.

Palavras-chave: plantas medicinais, fitoterapico, 1,2- benzopirona

\begin{abstract}
Validation of a rapid analytical method by HPLC for determining coumarin in guaco (Mikania glomerata Sprengel) confirmed with mass spectrometry. The species Mikania glomerata Sprengel, popularly known in Brazil as "guaco", is widely used as an expectorant to treat respiratory diseases. Its pharmacological activity is mainly attributed to coumarin. The results showed that the method for determining coumarin presented linearity from 0.05 to $0.8 \mathrm{mg} \mathrm{mL}-1$. It was considered selective, accurate, and precise according to the specific resolution from ANVISA, the Brazilian regulatory agency. The proposal of a rapid method for the determining coumarin in extracts of guaco is interesting for routine industrial quality control in order to obtain standardized, efficient, and safe phytotherapic medicines.
\end{abstract}

Keywords: medicinal plants, phytotherapic, 1,2- benzopyrone.

\section{INTRODUÇÃO}

A Mikania glomerata Sprengel, conhecida popularmente como guaco, é uma planta brasileira utilizada para fins medicinais e empregada principalmente como broncodilatadora e expectorante em tratamento de enfermidades do aparelho respiratório como tosse, bronquite e asma (Panizza, 1997; Moura et al., 2001; Corrêa et al., 2008) Indústrias de fitoterápicos produzem medicamentos à base de guaco e encontramse comercializados principalmente nas formas farmacêuticas de extrato, tintura e xarope (Santos, 2005). A maioria das plantas são obtidas por extrativismo na mata, possibilitando o uso incorreto de diferentes espécies do gênero Mikania na composição destes produtos. (Lorenzi \& Matos, 2002).

A cumarina é considerada a principal substância ativa do guaco, apresentando efeitos farmacológicos comprovados como espasmolítico, antiinflamatório e broncodilatador (Leite et al., 1993; Silva et al., 2008). Além disso, por ser um dos principais constituintes do vegetal é classificada também como marcador químico da espécie Mikania glomerata para controle de qualidade de formulações a base de guaco (Brasil, 2004). Estruturalmente são lactonas do ácido o-hidroxicinâmico, sendo o representante mais simples a cumarina (1,2-benzopirona), conforme mostra a (Figura 1). A cumarina é formada após lactonização do seu composto precursor, o ácido o-cumárico, por ação enzimática e na presença de calor, processos que ocorrem na danificação dos tecidos vegetais durante processamento das folhas de guaco para obtenção de extrato (Santos, 2005).

No Brasil, foi estabelecido o desenvolvimento de ações voltadas à implantação de plantas 


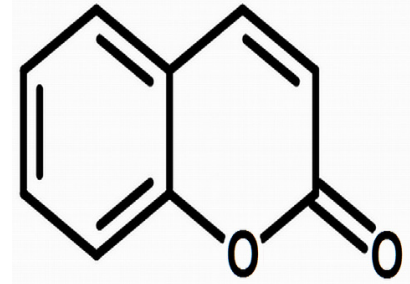

FIGURA 1. Estrutura química da cumarina $(1,2$ benzopirona)

medicinais e de fitoterápicos, visando garantir o monitoramento dos fitoterápicos pelo Serviço Nacional de Vigilância Sanitária. Isto porque, segundo a Resolução RE $n^{\circ} 89$ da Agência Nacional de Vigilância Sanitária (ANVISA), somente os fitoterápicos que fazem parte da Lista de Registro Simplificado de Fitoterápicos estão liberados para serem comercializados por empresas que possuam o certificado de boas práticas de fabricação e controle (Brasil, 2004). Dentre os fitoterápicos que fazem parte desta lista de registro da ANVISA está o guaco (Mikania glomerata Sprengel), sendo recomendado o uso de suas folhas nas formas de extrato fluído e tintura, padronizados em cumarina, indicados como expectorante e broncodilatador (Brasil, 2004; Farmacopéia Brasileira, 2005). Em virtude das propriedades terapêuticas atribuídas a essa espécie de planta, foi incluída no elenco de referência de medicamentos e insumos complementares para a assistência farmacêutica na atenção básica em saúde, conforme anexo II da Portaria 2.982 de 26 de novembro de 2009 (Brasil, 2009). Sendo assim, os medicamentos fitoterápicos a base de guaco vêm sendo utilizados em larga escala na rede de saúde pública pelo Sistema Único de Saúde (SUS), através da implantação de programas de fitoterapia em vários municípios nos estados brasileiro (Brasil, 2006). Visando o controle de qualidade e a padronização destes fitoterápicos, a ANVISA estabeleceu o marco regulatório para produção, distribuição e uso de plantas medicinais, particularmente sob a forma de drogas vegetais através da Resolução n`10 de 9 de março de 2010 (Brasil, 2010).

Apesar da ampla utilização de extratos de guaco em fitoterápicos, poucos são os métodos encontrados na literatura para a análise quantitativa de cumarina nesses extratos. No entanto, o controle de qualidade é baseado no teor de cumarina mínimo de 0,1 \% segundo a Farmacopéia Brasileira (2005) para guaco-cheiroso. Sendo assim, a quantificação de cumarina torna-se importante, já que se pode inferir sobre a qualidade da matériaprima vegetal utilizada na preparação, bem como na eficiência do método de extração empregado. Além disso, a quantificação de cumarina em guaco tem importância não somente devido às suas comprovadas atividades farmacológicas, mas também devido a efeitos colaterais em decorrência do uso excessivo, podendo causar vômitos, diarréias e hipertensão (Nolla \& Severo, 2005). Neste sentido, a recomendação da ANVISA é de que a dose diária máxima de cumarina seja de 4,89 mg de acordo com a lista de registro simplificado de fitoterápicos (Brasil, 2004).

Existem algumas técnicas para quantificação de cumarina e na sua maioria são realizadas por cromatografia líquida de alta eficiência (CLAE). No entanto, os procedimentos até então estabelecidos na literatura são relativamente demorados, variando de 10 a 20 min de tempo de análise, com fluxos que podem chegar a $1 \mathrm{~mL} \mathrm{~min}^{-1}$ de fase móvel (Celeghini et al., 2001; Santos, 2005; Castro et al., 2006; Alvarenga et al., 2009; Radünz et al., 2012; Melo \& Sawaya, 2015). Esse tempo de análise, associado à fluxos desta ordem, acabam onerando os custos de análise e geram resíduos químicos ao ambiente. Desta forma, este trabalho propõe estabelecer um método analítico rápido para determinar cumarina em extrato de guaco, contribuindo com o controle de qualidade deste fitoterápico através de um método analítico confiável, mas associado a ganho de tempo de análise e, consequentemente gerando menos resíduos no descarte da fase móvel, seguindo desta forma os princípios da química verde.

\section{MATERIAL E MÉTODO \\ Material vegetal e extração}

Foram utilizadas as folhas da espécie Mikania glomerata Spreng., coletadas no banco ativo de germoplasma (BAG) da Estação Experimental da Epagri de Itajaí, SC. Uma exsicata foi depositada no Herbário Barbosa Rodrigues (HBR), em Itajaí, SC, sob registro 54.403. O material vegetal foi desidratado a $40{ }^{\circ} \mathrm{C}$ em estufa com ventilação forçada durante $72 \mathrm{~h}$, moído e, a partir das amostras pulverizadas, foram preparados extratos por maceração: folhas secas em pó $(500 \mathrm{mg})$ foram macerados com etanol:água (1:1, v/v, $3 \mathrm{~mL})$ durante o periodo de 7 dias a temperatura ambiente com ausência de luz segundo metodologia recomendada pela Farmacopéia Brasileira (Silva, 1929). O material foi filtrado e o extrato foi analisado após diluído cinco vezes em fase móvel em sistema CLAE-UV.

\section{Reagentes}

Os solventes utilizados para as extrações das amostras foram grau analítico (Synth, São Paulo, Brasil). Para as análises cromatográficas utilizou-se: padrão de cumarina ( $\geq 99 \%$, Sigma, Missouri, USA), acetonitrila grau CLAE (Tedia, Rio de Janeiro, Brasil) e água ultrapurificada em sistema

Rev. Bras. PI. Med., Campinas, v.18, n.1, supl. I, p.316-325, 2016. 
Milli-Q (Millipore, São Paulo, Brasil).

Equipamento e condições cromatográficas

Foi utilizado cromatógrafo a líquido de alta eficiênciada, marca Agilent, modelo 1220 Infinity LC, dotado de bomba para gradiente binário, injetor automático com alça de amostragem de 10 $\mu \mathrm{L}$. Forno de coluna ajustado em $40^{\circ} \mathrm{C}$ e detector ultra violeta $(254 \mathrm{~nm})$. Utilizou-se coluna com fase estacionária octadecil, Luna C18(2) $3 \mu \mathrm{m} 30 \times 3$ $\mathrm{mm}$ (Phenomenex, California, USA); fase móvel água:acetonitrila $(55: 45 \mathrm{v} / \mathrm{v})$, modo isocrático e vazão $0,6 \mathrm{~mL} / \mathrm{min}$, com tempo total de eluição de 1 minuto. Os dados cromatográficos foram analisados usando-se o software do equipamento.

\section{Validação do método}

Os parâmetros avaliados foram linearidade, precisão (intermediária e repetibilidade), exatidão (recuperação), limite de detecção, limite de quantificação e seletividade.

A curva analítica foi obtida a partir de 6 pontos de soluções-padrão de cumarina preparados em triplicata em etanol $50 \%$ nas concentrações de 0,$05 ; 0,10 ; 0,20 ; 0,40 ; 0,60$ e $0,80 \mathrm{mg} \mathrm{mL}^{-1}$ a partir de uma solução estoque de $1,0 \mathrm{mg} \mathrm{mL}^{-1}$. A linearidade foi estimada pelo cálculo da equação de reta e o coeficiente de correlação linear nos intervalos de concentração avaliados.

O limite de detecção (LD) foi estimado através da relação de 3 vezes o ruído da linha de base (3:1), por diluições sucessivas do padrão de cumarina. O limite de quantificação (LQ) foi determinado através do ruído da linha de base e foi considerado como a concentração que produziu a relação sinal-ruído superior a 10:1.

A precisão do método foi avaliada em relação aos níveis de repetibilidade (precisão intradia) e precisão intermediária (precisão interdias). Para avaliar a repetibilidade analítica da metodologia empregada na análise, prepararam-se 7 amostras que foram injetadas em triplicata no mesmo dia, nas mesmas condições cromatográficas e pelo mesmo analista e os resultados foram expressos em coeficiente de variação (CV \%). Para a determinação da precisão intermediária foram preparadas para cada dia 7 amostras, que foram avaliadas em 3 dias diferentes por 3 analistas diferentes. As amostras foram injetadas em triplicata e os resultados expressos em CV \%.

A exatidão foi avaliada em termos de recuperação pelo método de adição padrão, uma vez que não é possível obter a matriz isenta do analito (cumarina). Sendo assim, quantidades conhecidas de cumarina foram adicionadas em triplicata no extrato fluído de guaco, obtendo três níveis de concentração: baixo $\left(0,25 \mathrm{mg} \mathrm{mL}^{-1}\right)$, médio $\left(0,40 \mathrm{mg} \mathrm{mL}^{-1}\right)$ e alto $\left(0,70 \mathrm{mg} \mathrm{mL}^{-1}\right)$. As amostras sem adição de padrão e com adição de padrão foram analisadas simultaneamente.

A recuperação foi expressa em termos de porcentagem obtida pelo valor da relação entre a concentração média determinada experimentalmente e a concentração teórica correspondente após a adição do analito na matriz, multiplicada por 100.

A seletividade de um método instrumental de separação é a capacidade de medir exatamente um composto em presença de outros componentes como impurezas, produtos de degradação e componentes da matriz (Brasil, 2003; Inmetro, 2010). A seletividade garante que o pico de resposta seja exclusivamente do composto de interesse, neste caso, o da cumarina (Ribani et al., 2004). O método de adição padrão foi aplicado para os estudos de seletividade através da elaboração de duas curvas analíticas, uma com as soluções de trabalho mistas adicionadas à matriz e a outra sem a presença da matriz. Comparando-se então as duas curvas analíticas, caso elas sejam paralelas, pode-se dizer que não há interferências de outros compostos da matriz na determinação da substância de interesse, portanto, o método é seletivo (Brasil, 2003). A seletividade foi também confirmada por espectrometria de massas (LC-ESI-MS) acoplado a um outro cromatógrafo líquido de alta eficiência (1290 Infinity Binary HPLC - Agilent Technologies, USA) com mesmas condições cromatográficas aplicadas no sistema CLAE-UV, com exceção da presença de $0,1 \%$ de ácido fórmico grau HPLC na fase móvel para auxiliar na protonação. Foi avaliado o padrão analítico de cumarina e duas amostras de extratos de guaco em modo scan e também em modo MS/MS para confirmação. A aquisição dos dados foi realizada em um equipamento Agilent Ifunnel Q-TOF 6550 com uma fonte de inozação Dual-AJS-ESI (Dual Agilent Jet Stream-ESI) no modo positivo com as seguintes condições: temperatura do gás de secagem $290{ }^{\circ} \mathrm{C}$; fluxo do gás de secagem de $11 \mathrm{~L} \mathrm{~min}^{-1}$; temperatura do gás de bainha de $325^{\circ} \mathrm{C}$, fluxo do gás de bainha de 10 L min-1; pressão de nebulização de 35 psi; Voltagem do capilar (Vcap) $3500 \mathrm{~V}$; voltagem de fragmentação $100 \mathrm{~V}$; Voltagem do OCT 1 RF Vpp $750 \mathrm{~V}$; e voltagem Nozzle $0 \mathrm{~V}$; energia de colisão com $\mathrm{N}_{2}$ de $20 \mathrm{~V}$ e faixa de aquisição do espectrômetro de massas para o experimento de MS entre 100 a $400 \mathrm{~m} / \mathrm{z}$ e para experimento de MS/MS com um íon alvo entre 50 a $150 \mathrm{~m} / \mathrm{z}$ (Grisi et al.,2015). Os resultados foram analisados usando-se o software do equipamento.

\section{RESULTADOS E DISCUSSÃO}

As curvas analíticas elaboradas por padronização externa para cumarina demonstraram

Rev. Bras. PI. Med., Campinas, v.18, n.1, supl. I, p.316-325, 2016. 
linearidade na faixa avaliada de 0,05 a 0,80 $\mathrm{mg} \mathrm{mL}^{-1}$ ( $\mathrm{n}=6$ pontos $)$ com coeficiente de correlação médio de 0,9925 . A equação da reta foi $y=21266 x+768,07$.

O limite de detecção (LD) obtido foi de 2,5 $\mu \mathrm{g} \mathrm{mL}-1$ e o limite de quantificação (LQ) foi considerado como 10 vezes a relação sinal ruído, ou seja $9,5 \mu \mathrm{g} \mathrm{mL}-1$. Esses resultados demonstram que o método é sensível para detectar e quantificar os níveis de concentração cumarina presentes em amostras de extrato de guaco.

A precisão do método (Tabela 1) foi avaliada através da repetibilidade de diversas medições realizadas num único dia com as mesmas condições cromatográficas e pelo mesmo analista. Já a precisão intermediária foi avaliada em 3 dias diferentes e por analistas diferentes.

Os valores do coeficiente de variação obtidos indicam uma precisão aceitável para a validação de um método analítico, uma vez que valores abaixo de $5 \%$ são considerados admissíveis de acordo com a ANVISA (2003).

A exatidão do método foi determinada por meio da obtenção da porcentagem de recuperação média, conforme descrito na Tabela 2. Para isso, o método de adição padrão foi utilizado, pois não é possível obter a matriz isenta do analito. Sendo assim, a eficiência de recuperação do método foi realizada através da adição de quantidades conhecidas de cumarina em quadruplicata nos extratos de guaco, em três níveis de concentração.

A recuperação ficou entre 98,38 e 107,98 $\%$, estando de acordo com as especificações esperadas para matrizes complexas (80-120 \%) (ANVISA, 2003). Os valores de coeficiente de variação ficaram entre 0,47 e 1,07 \%, estando abaixo de $2 \%$, conforme é recomendado por Green (1996).
Pode-se observar, conforme (Figura 2), que o método é seletivo quando se avalia a comparação das duas curvas analíticas: a primeira obtida de amostra de extrato fluído de guaco adicionados de padrões de cumarina ( $n=5$ pontos) e a segunda curva analítica obtida a partir de soluções padrões de cumarina ( $\mathrm{n}=5$ pontos).

O método de adição padrão foi aplicado para a avaliação de seletividade deste método, uma vez que não é possível obter a matriz (extrato de guaco) isenta de cumarina. Sendo assim, foi realizada uma curva analítica com adição da substância de interesse (cumarina) na matriz e comparada com uma curva analítica sem a presença da matriz (cumarina em etanol $50 \%$ ). Neste caso, observou-se que as duas curvas são paralelas com inclinação da reta semelhantes e com valores

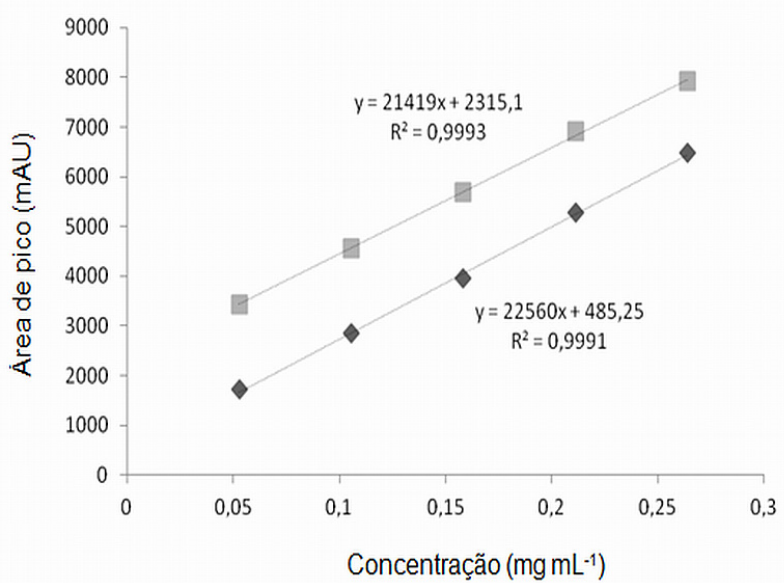

FIGURA 2. Curvas analíticas de padrões de cumarina em extrato de guaco ( $\square$ ) e dos padrões de cumarina em solução alcoólica $50 \%$ ( $\$$

TABELA 1. Valores das concentrações médias, desvios padrões e coeficientes de variações para avaliação de repetibilidade e precisão intermediária de método cromatográfico para determinação de cumarina

\begin{tabular}{ccc}
\hline & Repetibilidade & Precisão intermediária \\
\hline Média $\left(\mathrm{mg} \mathrm{mL}^{-1}\right)$ & 0,5002 & 0,5187 \\
Desvio Padrão & 0,0006 & 0,0215 \\
CV $(\%)$ & 0,1288 & 4,1384 \\
\hline
\end{tabular}

CV- coeficiente de variação.

TABELA 2. Porcentagem de recuperação de cumarina em três níveis de concentração em extrato fluído de guaco

\begin{tabular}{cccc}
\hline $\begin{array}{c}\text { Concentração estimada }(\mathbf{m g} \\
\mathbf{m L}-1)\end{array}$ & $\begin{array}{c}\text { Média concentração Experimental CLAE } \\
\left(\mathbf{m g ~ m L}^{-1}\right)\end{array}$ & $\begin{array}{c}\text { Recuperação } \\
(\%)\end{array}$ & $\begin{array}{c}\text { CV } \\
(\%)\end{array}$ \\
\hline 0,23 & 0,24 & 102,60 & 1,07 \\
0,41 & 0,45 & 107,98 & 0,85 \\
0,71 & 0,70 & 98,38 & 0,47 \\
\hline
\end{tabular}

CV- coeficiente de variação.

Rev. Bras. PI. Med., Campinas, v.18, n.1, supl. I, p.316-325, 2016. 

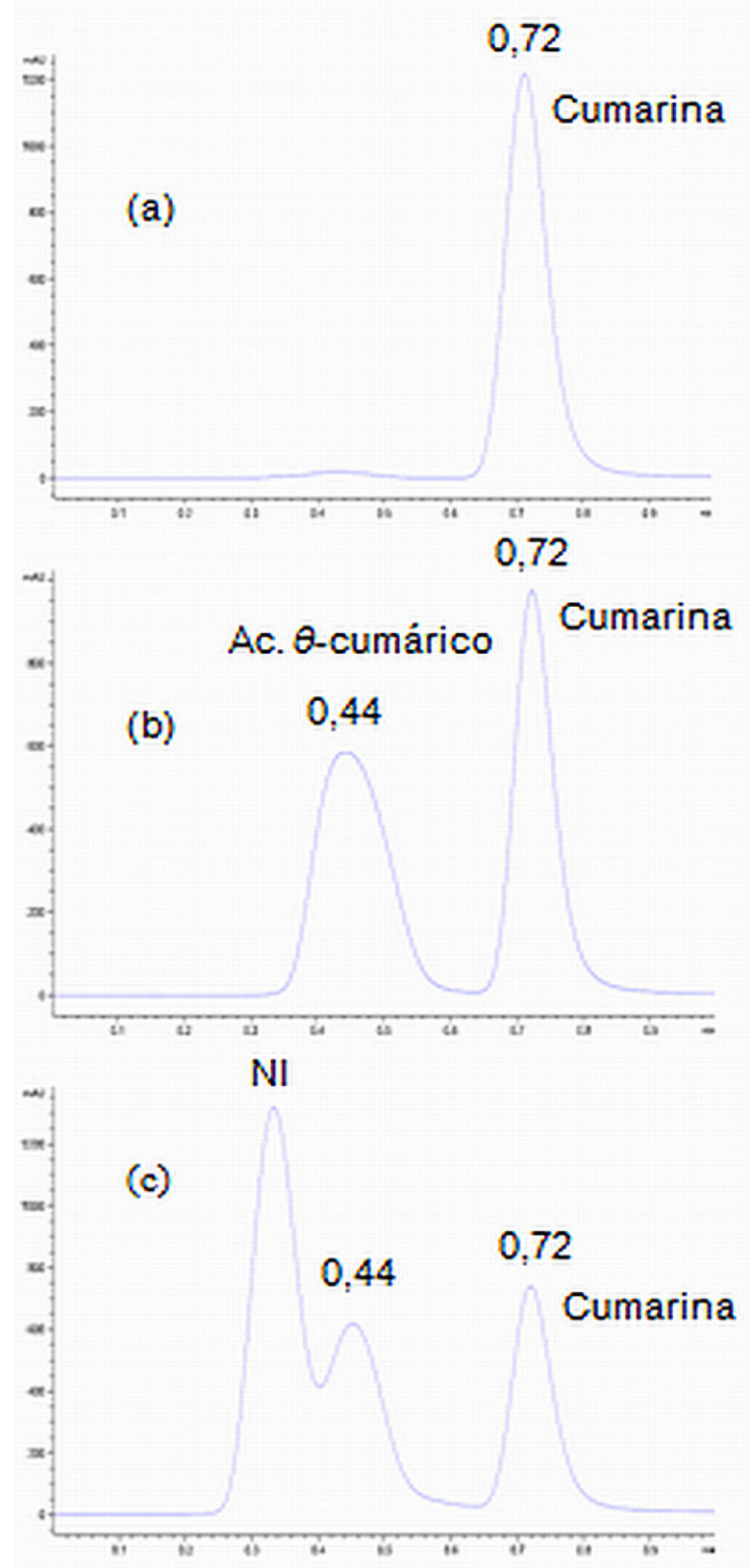

FIGURA 3. Cromatogramas: (a) padrão de cumarina $0,23 \mathrm{mg} \mathrm{mL}^{-1}$ preparada em etanol $50 \%$, (b) padrões de ácido o-cumárico e cumarina $\left(0,17 \mathrm{mg} \mathrm{mL}^{-1}\right)$ preparada em etanol $50 \%$, (c) extrato de guaco em solução alcoólica $50 \%$ com teor de cumarina determinada em $0,13 \mathrm{mg} \mathrm{mL}^{-1}, \mathrm{Nl}=$ não identificado

dos coeficientes angulares muito próximos (21419 e 22560) e com razão entre os dois coeficientes se aproximando de 1. Desta forma, pode-se observar que não houve interferência da matriz na determinação da cumarina, portanto o método demonstrou ser seletivo.

O processo de separação e determinação da cumarina pelo método proposto apresentou também boa seletividade quando avaliado em relação a separação do pico do analito de interesse (cumarina) aos demais compostos presentes na amostra. Como pode ser observado na Figura 3, o ácido o-cumárico, substância precursora da cumarina e quase sempre presente em amostras de extrato de guaco apresentou tempo de retenção (TR) de 0,44 min, não coeluindo desta forma com a cumarina (TR de 0,72 min), demonstrando a especificidade do método em relação ao analito citado.

Como pode ser observado na Figura 3, o tempo retenção da solução padrão de cumarina e na amostra foi o mesmo (0,72 min), indicando se tratar de um método extremamente rápido para sistemas de CLAE, com tempo de análise cromatográfica total de apenas $1 \mathrm{~min}$. Alguns poucos métodos analíticos para determinação de cumarina em guaco foram desenvolvidos e validados, entretanto nenhum até o momento foi descrito na literatura com tempo de análise de $1 \mathrm{~min}$. Conforme descrito por outros autores, o tempo de uma análise cromatográfica por CLAE para determinar cumarina pode variar de 10 a 20 min (Celeghini et al., 2001; Santos, 2005; Castro et al.,2006; Alvarenga et al., 2009; Radünz et al., 2012; Melo \& Sawaya, 2015). Esse tempo de análise, relativamente longo, se deve ao fato de serem utilizadas colunas C18 de $250 \mathrm{~mm}$ de comprimento.

Como substâncias diferentes podem apresentar respostas similares em dadas condições foi estabelecido proceder à análise por outra técnica comprobatória como a cromatografia líquida de alta eficiência acoplada à espectrometria de massas (LCESI-MS) para confirmar efetivamente a seletividade do método. A otimização do método foi a mesma utlizada no sistema validado em CLAE-UV, sendo o único diferencial a utilização de um detector de espectrometria de massas.

A partir de um padrão de cumarina foi possível determinar o tempo de retenção da mesma. A Figura 4, que apresenta a corrente total iônica em função do tempo de análise do padrão, revelando que, nas condições de análise, o tempo de retenção da cumarina está próximo a 0,85 minutos. Este tempo de retenção obtido neste sistema é levemente maior do que no sistema CLAE-UV (0,72 min) e se deve ao fato da maior distância percorrida pelo analito ("volume morto") entre a saída da coluna e o detector de espectrometria de massas.

A cumarina protonada tem razão massa/ carga $(\mathrm{m} / \mathrm{z})$ exata de 147,04406 , desta forma podese extrair o espectro de massas da cumarina a partir da corrente total iônica e comparar a massa media com a massa teórica. A Figura 5, apresenta o espectro de massas da cumarina em modo scan com um $\mathrm{m} / \mathrm{z}$ de 147,0443, acumulando-se a corrente 


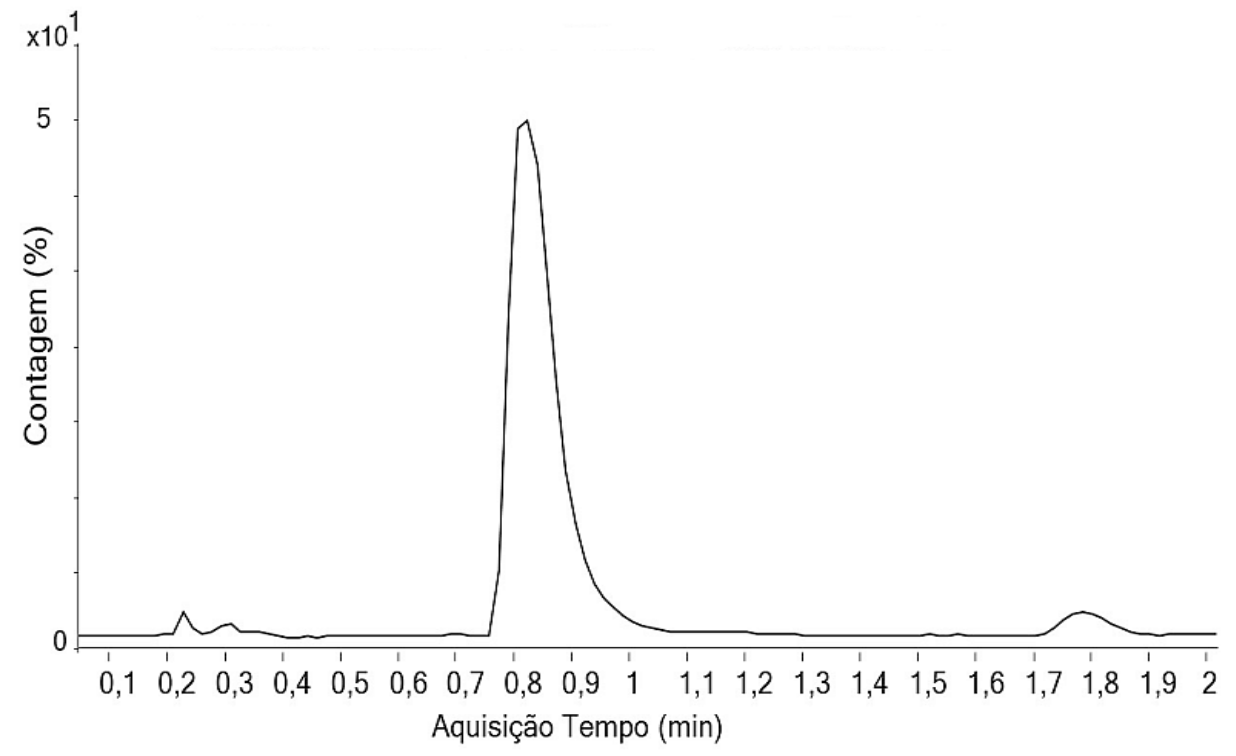

FIGURA 4. Corrente total iônica em função do tempo para um padrão de cumarina

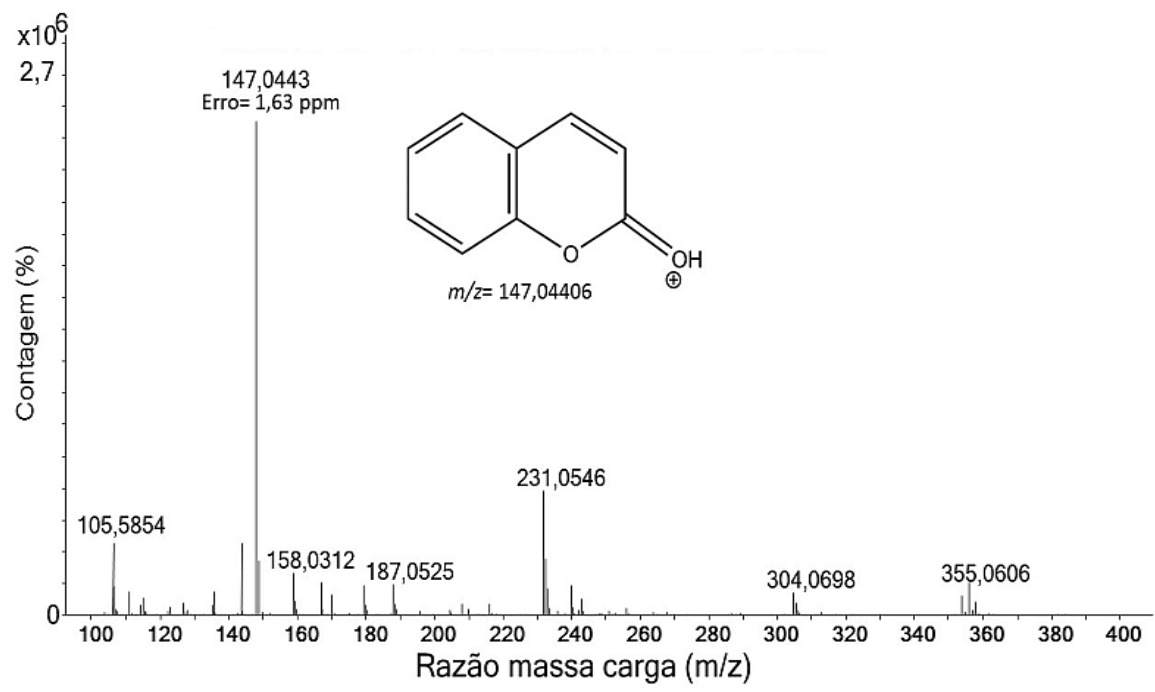

FIGURA 5. Espectro de massas do padrão de cumarina no modo scan (MS)

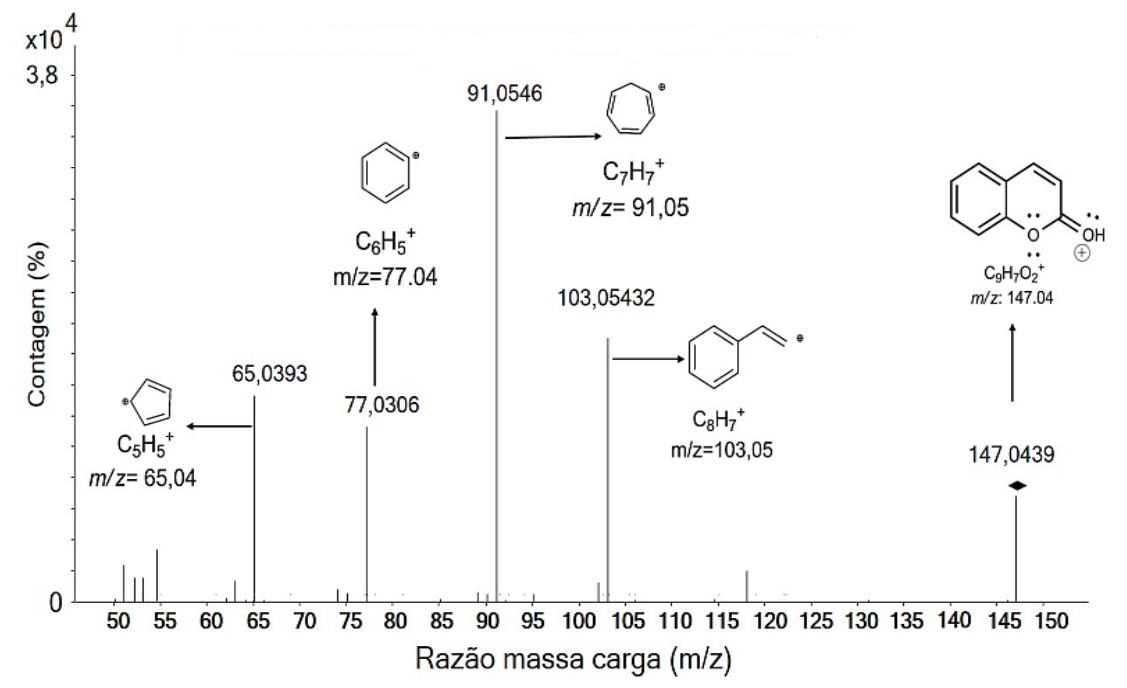

FIGURA 6. Espectro de massas da amostra padrão de cumarina no modo MS/MS

Rev. Bras. PI. Med., Campinas, v.18, n.1, supl. I, p.316-325, 2016. 


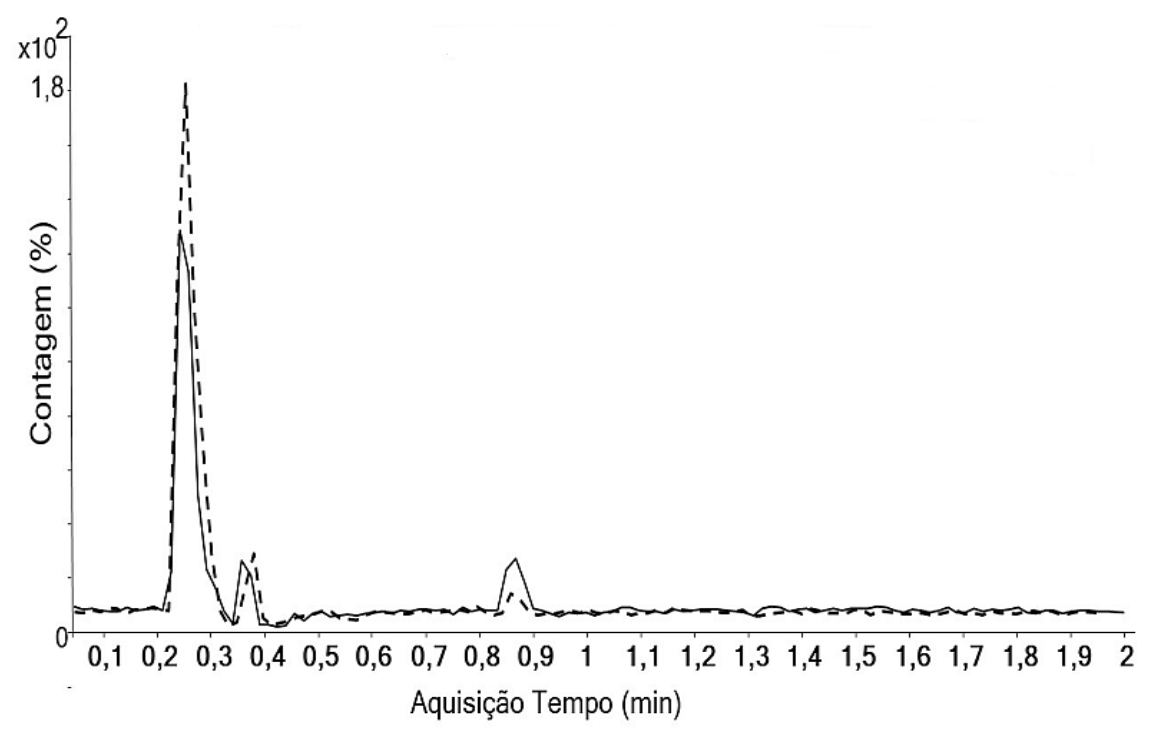

FIGURA 7. Corrente total iônica em função do tempo para as amostras 446 (- -) e 4410 (--)

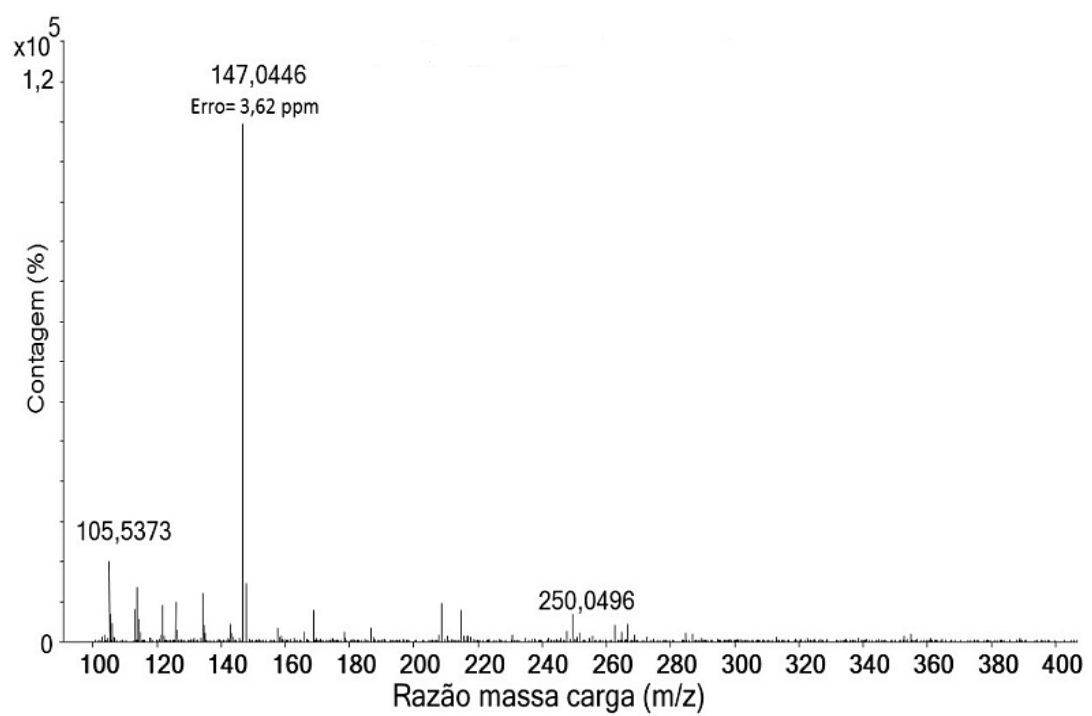

FIGURA 8. Espectro de MS de extrato de guaco da amostra 446

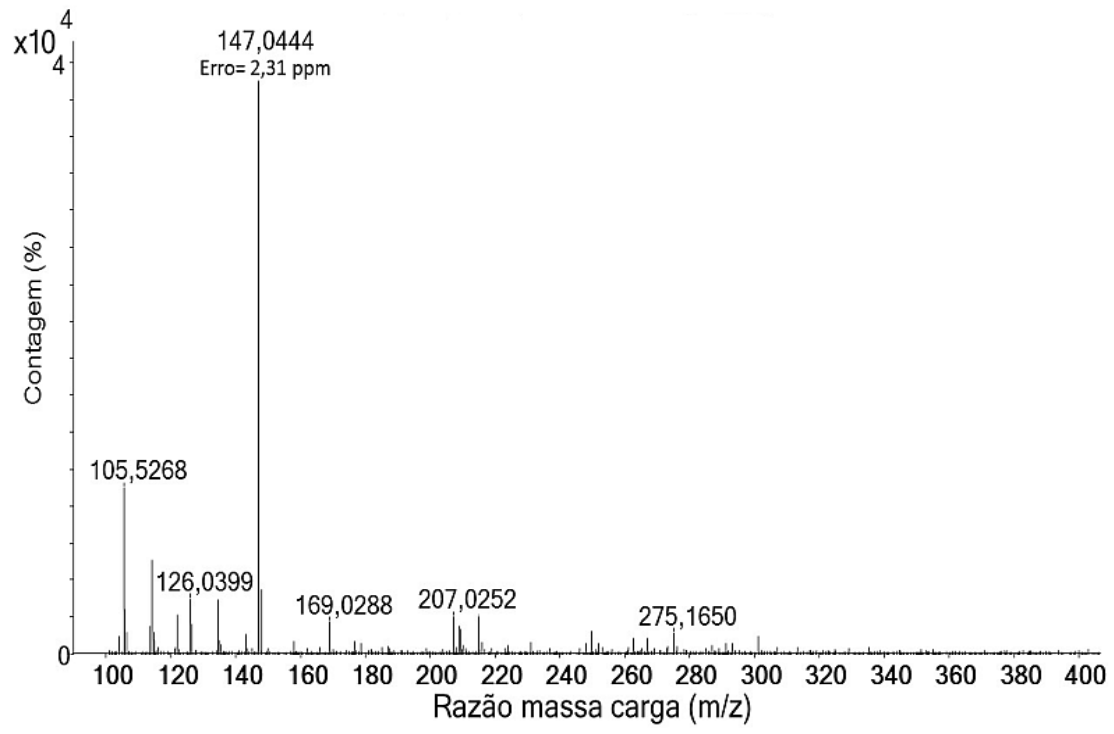

FIGURA 9. Espectro de MS da amostra de extrato de guaco 4410

Rev. Bras. PI. Med., Campinas, v.18, n.1, supl. I, p.316-325, 2016. 


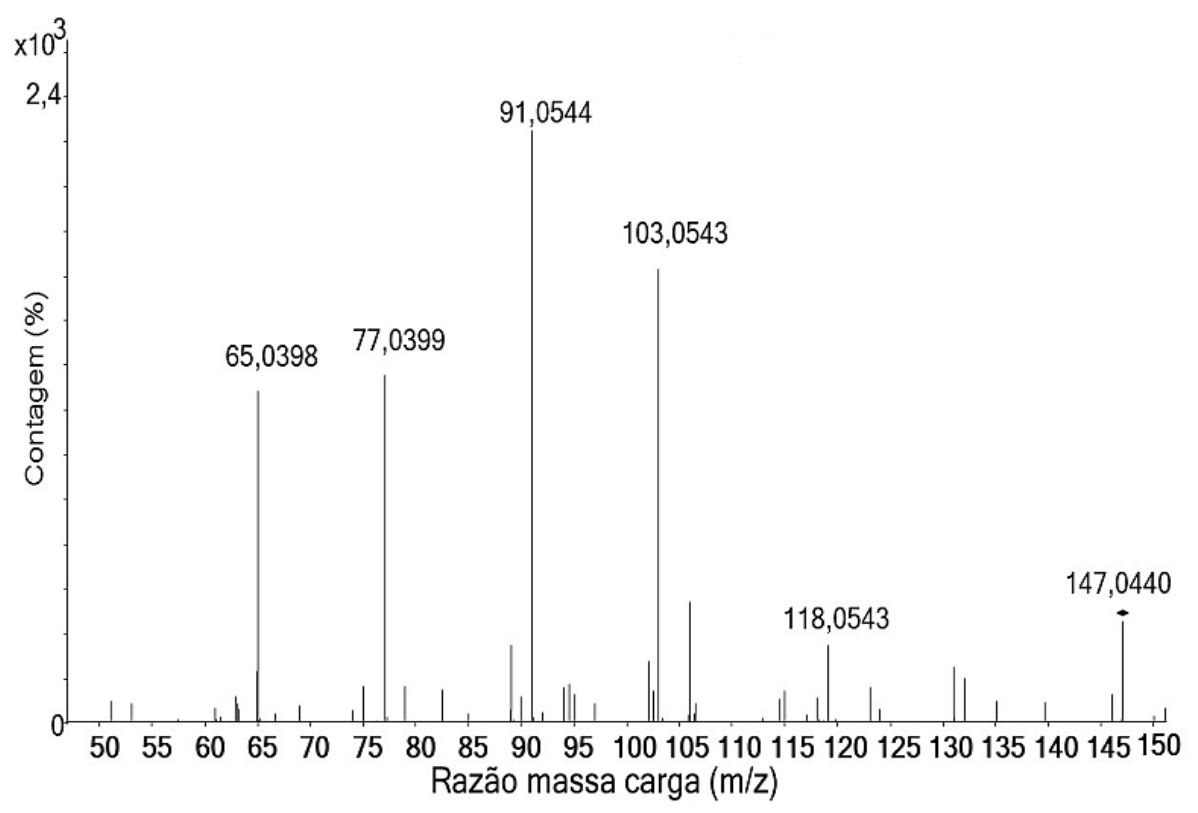

FIGURA 10. Espectro de MS/MS da amostra de extrato de guaco 446

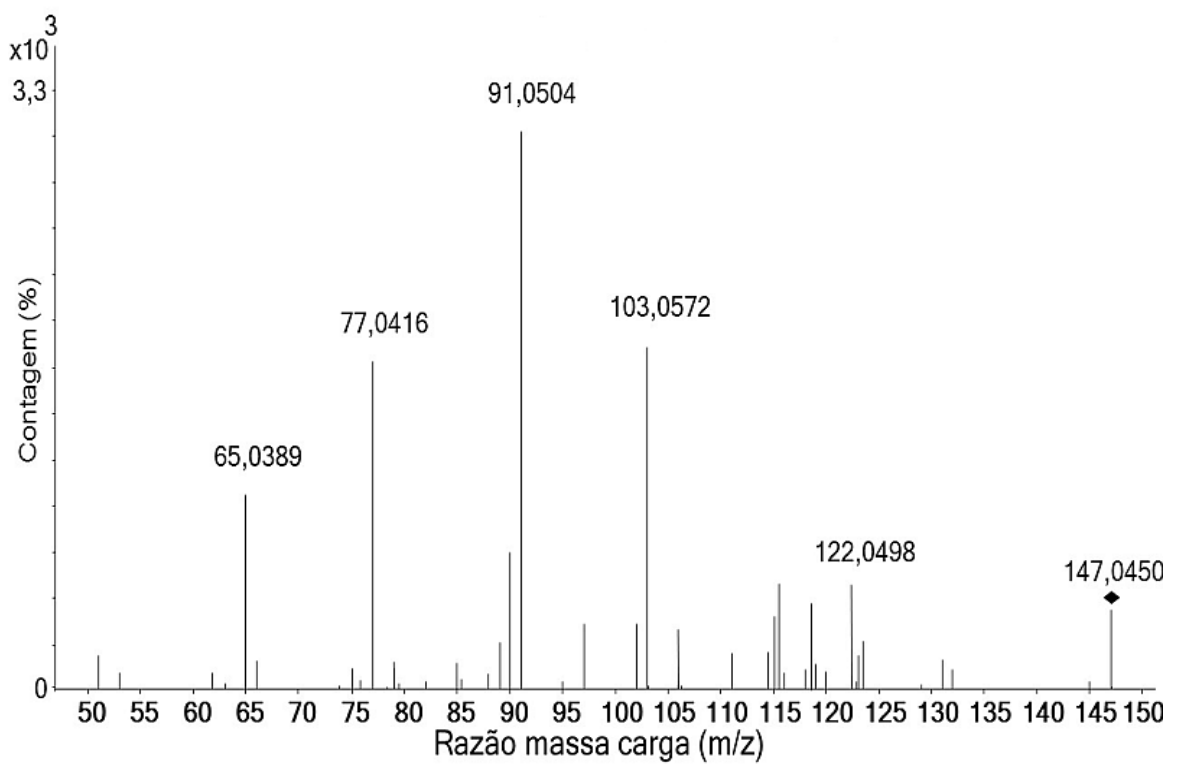

FIGURA 11. Espectro de MS/MS da amostra de extrato de guaco 4410

total iônica nas faixas de tempo de 0,80 a 0,90 minutos e subtraindo um branco extraído do intervalo de tempo de 1,3 a 1,4 minutos.

Posteriormente foi confirmado pelo modo de seleção MS/MS que o m/z de 147,0443 gerado no modo scan é, de fato, da mólecula de cumarina, uma vez que foi encontrado fragmentos com $\mathrm{m} / \mathrm{z}$ de 103,05432 formado pela perda neutra de $\mathrm{CO}_{2}$ da molécula, e os íons com $\mathrm{m} / \mathrm{z}$ de $91,0546,77,0306$ e 65,0393 que foram formados com rearranjo de anel aromático, conforme apresentado na Figura 6.

A partir de duas amostras de extrato de guaco denominadas de amostra 446 e amostra
4410 nas mesmas condições de análise foram três sinais de eluição para as duas amostras: o primeiro próximo a 0,25 minutos; o segundo próximo a 0,35 minutos e o ultimo em 0,85 minutos. A Figura 7, mostra a corrente total iônica para as duas amostras de extratos de guaco, acumulando-se a corrente total iônica nas faixas de tempo de 0,80 a 0,90 minutos e subtraindo um branco extraído do intervalo de tempo de 1,3 a 1,4 minutos para ambas as amostras.

A Figura 8 apresenta o espectro de MS da amostra 446 e na Figura 9 o da amostra 4410. Considerando que, os espectros no modo scan (MS) das duas amostras de extrato de guaco são 
semelhantes ao do espectro MS do padrão de cumarina (Figura 5), pode-se inferir que nenhum composto interferente desconhecido coeluiu juntamente no sinal da cumarina. Para confirmar que o íons gerados no modo scan das amostras de extrato de guaco são de fato da molécula de cumarina, foram realizados espectros de modo MS/MS das duas amostras, conforme mostram as Figuras 10 e 11.

\section{CONCLUSÃO}

Pode-se concluir que o método proposto é confiável para os parâmetros avaliados. As principais vantagens do método estariam relacionadas principalmente a redução do tempo de análise e da geração de resíduos, indo desta forma ao encontro dos princípios da química verde. Outra vantagem que vale ressaltar é com relação ao menor custo de aquisição da coluna, uma vez que colunas $\mathrm{C} 18$ de $30 \mathrm{~mm}$ de comprimento são menos onerosas quando comparada ao custo de colunas de 150 e $250 \mathrm{~mm}$.

A proposta de um método rápido tornase interessante para a rotina do controle de qualidade industrial, visando a obtenção de medicamentos fitoterápicos padronizados. Desta forma, o método desenvolvido e validado para análise de cumarina em extrato de guaco utilizando coluna de fase reversa de $30 \mathrm{~mm}$ atendeu às necessidades específicas do estudo, mostrando-se uma alternativa analítica rápida e eficiente.

\section{REFERÊNCIA}

ALVARENGA, F.C.R. et al. Avaliação da qualidade de amostras comerciais de folhas e tinturas de guaco. Revista Brasileira de Farmacognosia, v.19, p.442448, 2009.

BRASIL, Ministério da Saúde. Agência Nacional de Vigilância Sanitária (ANVISA). Resolução nº 899, de 29 de maio de 2003. Trata da validação de métodos analíticos e bioanalíticos, Brasília, 2003.

BRASIL, Ministério da Saúde. Agência Nacional de Vigilância Sanitária (ANVISA). Resolução n 89 de 16 Março de 2004. Determina a publicação da lista de registro simplificado de fitoterápicos, Brasília, 2004.

BRASIL, Ministério da Saúde. Conselho Nacional de Saúde. Portaria 971 de 03 de maio de 2006. Aprova a política nacional de políticas integrativas e complementares no sistema único de saúde (SUS), Brasília, 2006.

BRASIL, Ministério da Saúde. Portaria n² 2.982 de 26 de novembro de 2009. Aprova as normas de execução e de financiamento da assistência farmacêutica na atenção básica, Brasília, 2009.
BRASIL, Ministério da Saúde. Agencia Nacional de Vigilância Sanitária (ANVISA). Resolução $n^{\circ} 10$ de 9 março de 2010. Trata da notificação de drogas vegetais e dá outras providências, Brasília, 2010.

CASTRO E.M. et al. Coumarin contents in young Mikania glomerata plants (guaco) under different radiation levels and photoperiod. Acta Farmacéutica Bonaerense, v.25, p.387-392, 2006.

CELEGHINI, R.M.S. et al. Extraction and quantitative HPLC analysis of coumarin in hidroalchoolic extracts of Mikania glomerata Sprengel (guaco). Journal of the Brazilian Chemical Society, v.12, p.706-709, 2001.

CORRÊA, M.F.P. et al. Substâncias de origem vegetal potencialmente úteis na terapia da Asma. Revista Brasileira de Farmacognosia, v.18 (Supl.), p.785797, 2008.

FARMACOPEIA BRASILEIRA. 4.ed., II pt., 6 fasc., 2005. 305p. Disponível em: http://www.anvisa.gov.br/ hotsite/farmacopeiabrasileira/publicacoes/4_edicao/ parte2/4_edicao_fasc6.pdf. Acesso em: 19 abr. 2015.

GREEN, M.J. A practical guide to analytical method validation. Analytical Chemistry, v.68, n.9, p. 305309, 1996.

GRISI, P.U. et al. Phytotoxicity and Identification of Secondary Metabolites of Sapindus saponaria L. Leaf Extract. Journal of Plant Growth Regulation, v.34, p.339-349, 2015.

INSTITUTO NACIONAL DE METROLOGIA, NORMALIZAÇÃO E QUALIDADE INDUSTRIAL (INMETRO); Orientações sobre Validação de Métodos de Ensaios Químicos, DOQ-CGCRE-008, p.1-20, 2010. Disponível em: < http://www.inmetro. gov.br/Sidoq/Arquivos/CGCRE/DOQ/DOQCGCRE-8_03.pdf>. Acesso em: 19 abr. 2015.

LEITE, M.G.'. . et al. Estudo farmacológico comparativo de Mikania glomerata Sprengel (guaco), Justicia pectoralis Jacq (anador) e Torresea cearensis (cumaru). Revista Brasileira de Farmácia, v.74, n.1, p.12-15, 1993.

LORENZI, H.; MATOS, F. J. A. Plantas medicinais no Brasil: nativas e exóticas, 2. ed. Nova Odessa: Instituto Plantarum, 2002. 544p.

MELO, L.V. \& SAWAYA, A.C.H.F. UHPLC-MS quantification of coumarin and chlorogenic acid in extracts of the medicinal plants known as guaco (Mikania glomerata and Mikania laevigata). Revista Brasileira de Farmacognosia, v. 25, p.105-110, 2015.

MOURA, R.S. et al. Bronchodilator activity of Mikania glomerata Sprengel on human bronchi and guinea-pig trachea. Journal of Pharmacy and Pharmacology, v.54, p.249-256, 2001.

NOLLA, D. \& SEVERO, B. M. A. Plantas Medicinais. 2 ed., Passo Fundo: Editora Universitária UPF, 2005. $72 p$.

PANIZZA, S. Plantas que curam: cheiro de mato. 23. ed. São Paulo: IBRASA, 1997. 279 p.

RADÜNZ, L.L. et al. Rendimento extrativo de cumarina de folhas de guaco (Mikania glomerata Sprengel) submetidas a diferentes temperaturas de secagem. Revista Brasileira de Plantas Medicinais, v.14, n.3, p.453-457, 2012. 
RIBANI, M. et al. Validação em métodos cromatográficos e eletroforéticos. Química Nova, v.27, n.5, p.771780, 2004.

SANTOS, S. C.; Caracterização cromatográfica de extratos medicinais de guaco: Mikania laevigata Schulyz Bip. ex Baker e M. glomerata Sprengel e ação de $M$. laevigata na inflamação alérgica pulmonar. 2005. 93p. Dissertação (Mestrado em
Ciências Farmacêuticas) - Departamento de Farmácia, Universidade do Vale do Itajaí, Itajaí.

SILVA, R.A. Pharmacopeia dos Estados Unidos do Brasil, 1 ed., São Paulo: Nacional, 1929. 154p.

SILVA, C.R. et al. Método espectroscópico para determinação de cumarina em xarope de Mikania glomerata Sprengel. Revista Brasileira de Farmacognosia, v.18, p. 594-599, 2008. 